\title{
COX-2 silencing enhances tamoxifen antitumor activity in breast cancer in vivo and in vitro
}

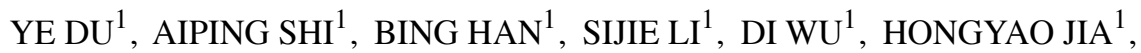 \\ CHAO ZHENG ${ }^{1}$, LIQUN REN $^{2 *}$ and ZHIMIN FAN ${ }^{1 *}$ \\ ${ }^{1}$ Department of Breast Surgery, The First Hospital, Jilin University; ${ }^{2}$ Department of Experimental Pharmacology \\ and Toxicology, School of Pharmacy, Jilin University, Changchun 130021, Jilin, P.R. China
}

Received December 3, 2013; Accepted January 16, 2014

DOI: 10.3892/ijo.2014.2299

\begin{abstract}
Tamoxifen (Tam), a selective estrogen receptor modulator, is in wide clinical use for the treatment and prevention of breast cancer. However, extended TAM administration for breast cancer induces increased VEGF levels in patients, promoting new blood vessel formation and thereby limiting its efficacy and highlighting the need for improved therapeutic strategies. Cyclooxygenase-2 (COX-2) silencing via a replication-incompetent lentivirus ( $\mathrm{LV}-\mathrm{COX}-2)$ induce cancer apoptosis and suppresses VEGF gene expression. In this study, the effect of LV-COX-2 infection, either alone or in combination with TAM, was analyzed in a breast cell lines for suppressing VEGF expression and simultaneously reducing doses of TAM. Cell proliferation, apoptosis, angiogenesis, metastasis, cell cycle distribution, an receptor signaling were determined after LV-COX-2 combination with TAM treatment. In addition, tumor growth ability in nude mice was detected to define the combination treatment effect in tumorigenesis in vivo. It is found that LV-COX-2 combination with TAM treatment in breast cancer cell significantly suppressed the proliferation and metastasis, and induced tumor apoptosis in vitro, and tumor growth also was suppressed in vivo. In addition, we also found that LV-COX-2 combination with TAM treatment could inhibit angiogenesis and VEGF expression. Taken together, our experimental results indicate that LV-COX-2 combination with TAM has promising outcome in anti-metastatic and apoptotic studies. Furthermore, these results showed that LV-COX-2
\end{abstract}

Correspondence to: Dr Liqun Ren, Department of Experimental Pharmacology and Toxicology, School of Pharmacy, Jilin University, Fujin Road, Changchun 130021, Jilin, P.R. China

E-mail: renliqun513@sina.com

Professor Zhimin Fan, Department of Breast Surgery, The First Hospital, Jilin University, Changchun 130021, Jilin, P.R. China E-mail: fanzhimin780213@126.com

${ }^{*}$ Contributed equally

Key words: breast cancer, tamoxifen, COX-2 silencing, antitumor activity combination with TAM is a potential drug candidate for treatment of breast tumors expressing high levels of VEGF.

\section{Introduction}

Breast cancer is the most common female cancer and $~ 70-75 \%$ of cases express oestrogen receptor $\alpha(\mathrm{ER} \alpha)$ (1). Tamoxifen (TAM), a selective estrogen receptor (ER) modulator, has been used extensively in the clinical management of primary and advanced breast cancer and is also widely employed as a preventive agent after surgery for breast cancer (2). A larger number of clinical studies over the past 30 years have shown that TAM can reduce the incidence and regression of breast carcinoma among women worldwide (3). Despite the relative safety and significant anti-neoplastic activities of tamoxifen, most initially responsive breast tumors develop resistance to its (4). In addition, TAM is also known to increase the expression of vascular endothelial growth factor (VEGF), which is an undesirable effect in breast cancer treatment $(5,6)$. Extensive studies have shown that VEGF, a receptor tyrosine kinase, plays an important role in tumorigenesis, and blocking the VEGF signaling pathway can reduce tumor-associated angiogenesis and blood vessel-dependent metastasis $(7,8)$. Therefore, combination therapies of tamoxifen with an antiVEGF signaling agent aimed at inhibition of both ER-mediated signaling and VEGF stimulated stromal activation, may be a potential means of delaying the arrival of resistance.

Cyclooxygenase-2 (COX-2) is an inducible gene, whose expression is undetectable in most normal tissues. Accumulating evidence shows that overexpression of COX-2 was commonly related to different types of carcinomas, including breast carcinoma (9-12). COX-2 has been implicated in breast tumorigenesis based on its increased expression in a significant fraction of breast carcinomas and the protective effects of nonsteroidal anti-inflammatory drugs (NSAIDs) against breast cancer (13). Hoeben et al (14) have indicated that COX-2 overexpression is correlated with induction of VEGF expression and therefore tumor angiogenesis in human breast cancers. Inhibition of COX-2 by non-steroidal antiinflammatory drugs (NSAD) leads to restricted angiogenesis and downregulates production of VEGF (15). Although accumulating evidence suggests that NSAIDs, such as celecoxib sensitize cancer cells to ATM-induced apoptosis, it remains 
unclear whether it is COX-2 dependent or independent (16). Furthermore, NSAIDs could result in liver injury, which would limit their clinical applications $(17,18)$.

Silencing of COX-2 by RNAi would avoid the side effects induced by NSAIDs. Accumulating evidence shows that downregulation of COX-2 expression by RNAi inhibited tumor cell proliferation and colony formation in vitro in different types of cancer cells $(12,19,20)$. COX-2 silencing abolished the metastatic potential of highly malignant breast cancer cells (10). In addition, Lin et al documented that combining gene therapy with various drugs enhances transduction efficiency resulting in enhanced tumor cell killing (21). Therefore, in this study, we knocked out the COX-2 gene via a replication-incompetent lentivirus (LV-COX-2), then LV-COX-2 combination with TAM for suppressing VEGF expression and simultaneously reducing doses of ATM.

The objective of the present study was to evaluate the potency of LV-COX-2 in combination with TAM in inhibiting breast cancer cell growth, proliferation, and angiogenesis in vitro and reveal the underlying molecular mechanisms involved in TAM-induced apoptosis. In addition, tumor growth ability in nude mice was detected to define the combination treatment effect in tumorigenesis in vivo.

\section{Materials and methods}

Cell culture. Human breast cancer cell lines MCF-7 and HEK293 (human embryonic kidney cells) were bought from the Shanghai Cell Collection (Shanghai, China). Cells were cultured in Dulbecco's modified Eagle's medium (DMEM; Gibco-BRL, Grand Island, NY, USA) supplemented with $10 \%$ heat-inactivated fetal bovine serum (FBS; Gibco-BRL) at $37^{\circ} \mathrm{C}$ in a $5 \% \mathrm{CO}_{2}$ atmosphere and at $95 \%$ humidity.

Reagents. Stock solutions of $10 \mathrm{mM}$ TAM (Sigma-Aldrich, St. Louis, MO, USA) were dissolved in dimethylsulfoxide (DMSO, Sigma-Aldrich), stored at $-20^{\circ} \mathrm{C}$, and diluted in fresh medium just before use. TRIzol reagent kit and Coomassie Blue R-250 from Gibco-BRL, Invitrogen Corp. (Carlsbad, CA, USA); Nonidet P-40 lysis buffer, chemiluminescent peroxidase substrate, propidium iodide (PI), 4',6-diamidino2-phenylindole (DAPI), 3-(4,5-dimethylthiazol-2-yl)-2,5 diphenyltetrazolium bromide (MTT), and sense and antisense VEGFR2 oligo primers from Sigma-Aldrich; and pyrogallol and $\mathrm{H}_{2} \mathrm{O}_{2}$ from Merck (Whitehouse Station, NJ, USA). Stock solutions of PI, DAPI, and MTT were prepared by dissolving $1 \mathrm{mg}$ of each compound in $1 \mathrm{ml}$ of phosphate buffered saline (PBS). The solution was protected from light, stored at $4^{\circ} \mathrm{C}$, and used within 1 month.

Animals. Female BALB/c nude mice aged 4-5 weeks were obtained from the Experimental Animal Center of the Jilin University (Changchun, China). All procedures were performed according to institutional guidelines and conformed to the National Institutes of Health guidelines on the ethical use of animals.

Generation of plasmids and recombinant lentivirus. To inhibit the expression of COX-2, a short hairpin RNA (shRNA) targeting the COX-2 transcript was designed. The synthesized oligonucleotides containing specific target sequence, a loop, the reverse complement of the target sequence, a stop codon for U6 promoter and two sticky ends were cloned into pGCSIL-GFP lentivirus vector according to the instructions (ShanghaiGene Chem Co.Ltd., China). The targeting sequences corresponding to the siRNAs for COX-2 (GeneBank accession no. NM_000963.2) was as followed: bases on 290-310 (sense 5'-AAACTGCTCAACACCGGAATT-3'). Sequences for the scrambled control (NC) for siRNA are AATTCTCCGAACG TGTCACGT (sense). This sequence does not target any gene product and have no significant sequence similarity to human gene sequences, being essential for determining the effects of siRNA delivery.

To produce the lentivirus, the HEK293 cells were transfected with $20 \mu \mathrm{g}$ of LV-COX-2, and LV-NC plasmid together with $15 \mu \mathrm{g}$ of pHelper-1.0 and $10 \mu \mathrm{g}$ of pHelper-2.0 packaging plasmids, respectively (22). The culture medium was collected within $48 \mathrm{~h}$ after transfection, concentrated by ultracentrifugation, aliquoted, and stored at $-80^{\circ} \mathrm{C}$ until used. The titer of lentivirus was determined by hole-by-dilution titer assay as described (23). Four days after a single exposure of HEK293 cells to the lentivirus, strong green fluorescence was observed in $>90 \%$ of cells, indicating a high and stable transduction of the lentiviral vector system. The final titer of LV-COX-2, and LV-NC were $5 \times 10^{8} \mathrm{TU} / \mathrm{ml}$ and $4 \times 10^{8} \mathrm{TU} / \mathrm{ml}$, respectively.

RNA isolation and real-time RT-PCR. Total RNA was extracted from cultured cells using TRIzol reagent (Invitrogen) according to the manufacturer's instructions. RNA was reverse-transcribed into cDNA by a Primescript ${ }^{\mathrm{TM}}$ RT reagent kit according to the manufacturer's protocols (Takara, Japan). Quantitative real-time polymerase chain reaction (RT-PCR) assays were carried out using SYBR Green Real-Time PCR Master Mix (Toyobo, Osaka, Japan) and RT-PCR amplification equipment using specific primers: COX-2, sense strand 5'-CCCTTGGG TGTCAAAGGTAAA-3', antisense strand 5'-AAACTGATGC GTGAAGTGCTG-3', $\beta$-actin, sense strand 5'-GCGAGCACA GAGCCTCGCCTTTG-3', antisense strand 5'-GATGCCGTG CTCGATGGGGTAC-3'. The PCR conditions were as follows: a pre-denaturing at $95^{\circ} \mathrm{C}$ for $3 \mathrm{~min}$, followed by 40 cycles of denaturation at $95^{\circ} \mathrm{C}$ for $10 \mathrm{sec}$, annealing/extension at $58^{\circ} \mathrm{C}$ for $20 \mathrm{sec}$, final extension of $72^{\circ} \mathrm{C}$ for $10 \mathrm{~min}$. The amplification specificity was checked by melting curve analysis. The $2^{-\Delta \Delta C T}$ method was used to calculate the relative abundance of target gene expression generated by Rotor-Gene Real-Time Analysis Software 6.1.81. The expression of interest genes were determined by normalization of the threshold cycle $(\mathrm{Ct})$ of these genes to that of the control $\beta$-actin.

Western blot analysis. Cultured cells were washed twice with phosphate-buffered saline (PBS, $\mathrm{pH} 7.2$ ), then cells were lysed with Triton X-100 in HEPES buffer $(150 \mathrm{mM} \mathrm{NaCl}, 50 \mathrm{mM}$ HEPES, $1.5 \mathrm{mM} \mathrm{MgCl}$, $1 \%$ Triton $\mathrm{X}-100,0.1 \%$ SDS, protease inhibitor cocktail (Sigma), $100 \mathrm{mM} \mathrm{NaF}$ and $100 \mathrm{mM} \mathrm{Na} \mathrm{VO}_{4}$ ) for $30 \mathrm{~min}$. Cell lysates were clarified by centrifugation (10,000 g, $15 \mathrm{~min})$, and protein concentrations were determined using the Bradford reagent (Sigma, Germany). Protein samples were separated on an 8-15\% SDS-polyacrylamide gel (SDS-Page) and transferred onto nitrocellulose membranes (Santa Cruz Biotechnology, Inc., Santa Cruz, CA, USA), 
followed by blocking in TBS containing 5\% skim milk and $0.1 \%$ Tween-20 for $2 \mathrm{~h}$ at room temperature, and then immunoblotted with specific primary antibodies and incubated with corresponding horseradish peroxidase-conjugated secondary antibody. The other primary antibodies used in the western blot analyses were: anti-VEGF, anti-VEGFR, anti-COX-2 (all Cell Signaling Technology, Beverly, MA, USA), mouse monoclonal anti- $\beta$-actin (Sigma-Aldrich), and mouse monoclonal anti-caspase-3, mouse monoclonal anti-caspase-8 (Santa Cruz Biotechnology). Secondary Abs used for immunodetection were: HRP-conjugated goat anti-mouse IgG and goat antirabbit IgG (Amersham Biosciences, Uppsala, Sweden). All immunoblots were visualized by enhanced chemiluminescence (Pierce).

Proliferation assays. To measure the effect of LV-COX-2 and ATM alone or both on cell proliferation, 3-(4,5-dimethylthiazol-2-yl)-2,5-diphenyltetrazolium bromide (MTT) assay was used. MCF-7 cells grown in monolayers were harvested and dispensed in 96-well culture plates in $100 \mu \mathrm{l}$ of DMEM at a concentration of $5 \times 10^{3}$ cells per well. After $24 \mathrm{~h}$, cells without any treatment, cells treated only with LV-NC, and cells transfected with LV-COX-2, cells treated only with ATM, cells treated with ATM combination with LV-COX-2 were seeded in 96-well plates and left at $37^{\circ} \mathrm{C}$ for $48 \mathrm{~h}$. The cells were then washed with PBS and incubated in $50 \mu 1$ of $0.5 \mathrm{mg} / \mathrm{ml}$ MTT in culture medium at $37^{\circ} \mathrm{C}$ for $4 \mathrm{~h}$. Following the addition of $100 \mu \mathrm{l}$ of isopropanol, the absorbance was read at $595 \mathrm{~nm}$ in an ELISA plate reader (Molecular Devices Corp., Sunnyvale, CA, USA). The mean proliferation of cells without any treatment was expressed as $100 \%$.

Detection of apoptosis. MCF-7 cells were cultured in 6-well plates in DMEM with $10 \%$ FBS medium and were treated with LV-NC, LV-COX-2, ATM and ATM combination with LV-COX-2, respectively, for $48 \mathrm{~h}$. The cover slips were washed three times with phosphate-buffered saline (PBS) and single cell suspensions were fixed in $1 \%$ PBS. Cells were stained with $100 \mu \mathrm{g} / \mathrm{ml}$ acridine orange (AO) and $100 \mu \mathrm{g} / \mathrm{ml}$ ethidium bromide (EB) for $1 \mathrm{~min}$. Then cells were observed under a fluorescence microscope. At least 200 cells were counted and the percentage of apoptotic cells was determined. In addition, we also detected caspase- 3 and -8 protein expression by western blotting as an additional indicator of apoptosis.

Cell cycle analysis. To determine the cell cycle distribution, $5 \times 10^{5} \mathrm{MCF}-7$ cells were plated in $60-\mathrm{mm}$ dishes and treated with LV-NC, LV-COX-2, ATM and ATM combination with LV-COX-2, respectively, for $48 \mathrm{~h}$. After treatment, the cells were collected by trypsinization, fixed in $70 \%$ ethanol, and kept at $-20^{\circ} \mathrm{C}$ overnight for fixation. Cells were washed in PBS, resuspended in $1 \mathrm{ml}$ of PBS containing $100 \mu \mathrm{g} / \mathrm{ml}$ RNase and $40 \mu \mathrm{g} / \mathrm{ml}$ PI and incubated in the dark for $30 \mathrm{~min}$ at room temperature. The distribution of cells in the cell cycle phases were analyzed from the DNA histogram with a FACSCaliber flow cytometer (Becton-Dickinson, San Jose, CA, USA) and CellQuest software (CA, USA).

Cell migration assay. The migration assay was performed using a 12-well Boyden chamber (Neuro Probe) with an 8- $\mu \mathrm{m}$ pore size. Approximately $1 \times 10^{5}$ cells were seeded into upper wells of the Boyden chamber and incubated for $6 \mathrm{~h}$ at $37^{\circ} \mathrm{C}$ in medium containing 1\% FBS. Medium containing 10\% FBS was used as a chemoattractant in the bottom wells. Cells that did not migrate through the pores of the Boyden chamber were manually removed with a rubber swab. Cells that migrated to the lower side of the membrane were stained with hematoxylin and eosin and photographed using an inverted microscope.

Wound-healing assay. To assess the effect of TAM and LV-COX-2 on cell migration, wound-healing assay was performed. MCF-7 cells $\left(1 \times 10^{5}\right)$ were plated in 12-well plates in complete growth medium. After $24 \mathrm{~h}$ of growth, a scratch was made through the confluent cell monolayer, and then the cells were treated with LV-COX-2, LV-NC, TAM, TAM combination with LV-COX-2, respectively, in $3 \mathrm{ml}$ of complete medium. At $48 \mathrm{~h}$ post-treatment, cells were stained with hematoxylin and eosin (HE). Cells invading the wound line were observed under an inverted phase-contrast microscope using 20x, Leica DMR, Germany.

Cell invasion assay. Cell invasion was determined using transwell chambers made from polycarbonate membrane filters with a pore size of $8 \mu \mathrm{m}$. Transwell filters in 6-well plates were coated with Matrigel, hydrated for $\sim 2 \mathrm{~h}$ in the tissue culture incubator with $500 \mu \mathrm{l}$ serum-free culture media in the bottom and $500 \mu \mathrm{l}$ in the top of the chamber. After hydration of the Matrigel, $5 \times 10^{5}$ MCF-7 cells were plated in $500 \mu \mathrm{l}$ serum-free medium on top of chamber, while $2 \mathrm{ml}$ medium $10 \%$ FCS were placed in the lower chambers. TAM, LV-NC, LV-COX-2, LV-COX-2 combination with ATM were added to the upper chambers, respectively. Cells without any drug were used as control. After $48 \mathrm{~h}$ of incubation, the filters were removed, washed twice in PBS and fixed in $10 \%$ formalin for $15 \mathrm{~min}$. After fixing at room temperature, the chambers are rinsed in PBS and stained with $0.2 \%$ crystal violet staining solution for $30 \mathrm{~min}$. After washing the chambers by PBS, the cells at the top of the Matrigel membrane were carefully removed by cotton swabs. At this time all cells that remain had invaded to the bottom side of the membrane. Cell invasion was observed with an immunofluorescence microscope by counting the cells that had invaded into the bottom of the Cell Culture Insert. All experiments were performed in triplicate.

Measurement of prostaglandin-E2 (PGE2) production. PGE2 synthesis was determined by competitive enzyme-linked immunosorbent assay (ELISA) as previously described (24). In brief, MCF-7 cells were treated with LV-NC, LV-COX-2, ATM and ATM combination with LV-COX-2 respectively for $48 \mathrm{~h}$ in 12-well plates, and then these culture media were centrifuged to remove cell debris. Cell-free culture media were collected at indicated time, and then PGE2 levels were determined by competitive ELISA as described using the kit manufacturer (Cayman Chemical, Ann Arbor, MI, USA).

Measurement of VEGF levels. To measure VEGF levels, $5 \times 10^{5}$ MCF-7 cells were plated in 6-well plates and incubated under culture conditions overnight, and the medium was replaced by serum-free culture conditioned medium. LV-NC, 
LV-COX-2, ATM and ATM combination with LV-COX-2, respectively, were added to the culture, and the medium was collected at $72 \mathrm{~h}$. VEGF levels were measured using a VEGF enzyme linked immunosorbent assay (ELISA) kit (DVE00, R\&D Systems, Minneapolis, MN, USA) according to the manufacturer's instructions. The optical density at $570 \mathrm{~nm}$ of each well was measured using an ELISA reader ( $\mu$ Quant; Biotek Instruments, Inc., Winooski, VT, USA). In addition, we also detected VEGF and VEGFR protein expression level by western blotting.

Tumor xenograft in nude mice. Exponentially growing MCF-7 cells were harvested and a tumorigenic dose of $2 \times 10^{6}$ cells was injected intraperitoneally into 4-5-week-old female BALB mice. When tumors reached $100-200 \mathrm{~mm}^{3}$, mice were divided randomly into five groups (10 mice per group). The control group received $1 \%$ polysorbate resuspended in deionized water. The other four groups were treated with LV-NC $\left(1 \times 10^{8} \mathrm{PFU} /\right.$

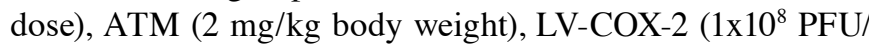
dose), or ATM plus LV-COX-2 (ATM, $1 \mathrm{mg} / \mathrm{kg}$ body weight; LV-COX-2, $1 \times 10^{8}$ PFU/dose respectively) intraperitoneally on alternative days for 3 weeks. The tumor size was measured using caliper before the treatment injections were given, and on day 7, 14 and 21 of treatment. On day 21, the animals were euthanized using chloroform and their spleen tissues were collected and cultured for a splenocyte surveillance study.

Assay of splenocyte proliferation. Spleens from treated mice were collected, and single-cell spleen suspensions were pooled in serum-free RPMI-1640 by filtering the suspension through a sieve mesh with the aid of a glass homogenizer to exert gentle pressure on the spleen fragments. The detail assay of splenocyte proliferation was previously described (16).

Statistical analysis. Data from at least three independent experiments are expressed as mean \pm SD. Statistical comparison of more than two groups was performed using one-way ANOVA followed by a Tukey post hoc test. Statistical analyses were undertaken using GraphPad Prism version 5.01 (GraphPad Software, San Diego, CA, USA) and the SPSS ${ }^{\circledR}$ statistical package, version 16.0 (SPSS Inc., Chicago, IL, USA) for Windows ${ }^{\circledR} . P<0.05$ was considered statistically significant.

\section{Results}

Downregulation of COX-2 expression by $L V$-COX-2. To silence COX-2 expression, we constructed a recombinant lentiviral carrying a based shRNA against COX-2, LV-COX-2, to inhibit COX-2 expression in breast carcinoma cell lines. To evaluate the silencing capacity of LV-COX-2, MCF-7 were treated with LV-COX-2, LV-NC as negative controls. Real-time RT-PCR and western blotting were performed to detect the COX-2 mRNA expression and protein expression levels at 3 days posttransfection. Our results showed no significant inhibition in COX-2 mRNA expression in LV-NC group, there is no significantly difference in LV-NC group and control group (PBS group) $(\mathrm{P}>0.05)$. On the other hand, COX-2 mRNA expression in LV-COX-2 group was significantly decreased compared to LV-NC group and control group after injection (Fig. 1A, p<0.01). On protein level, there was no significant

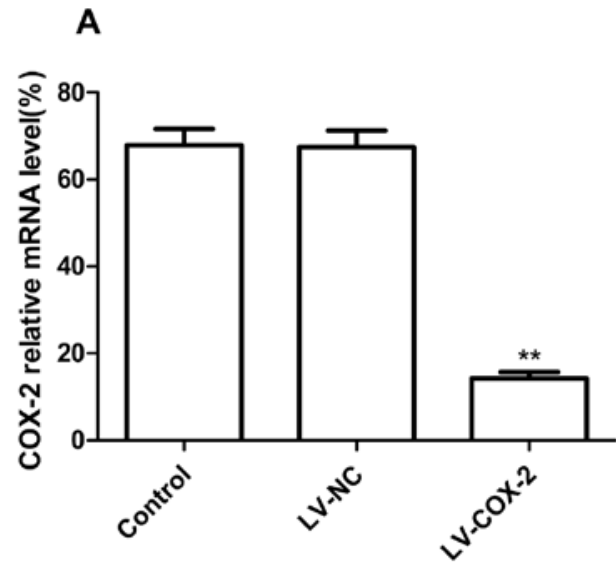

B

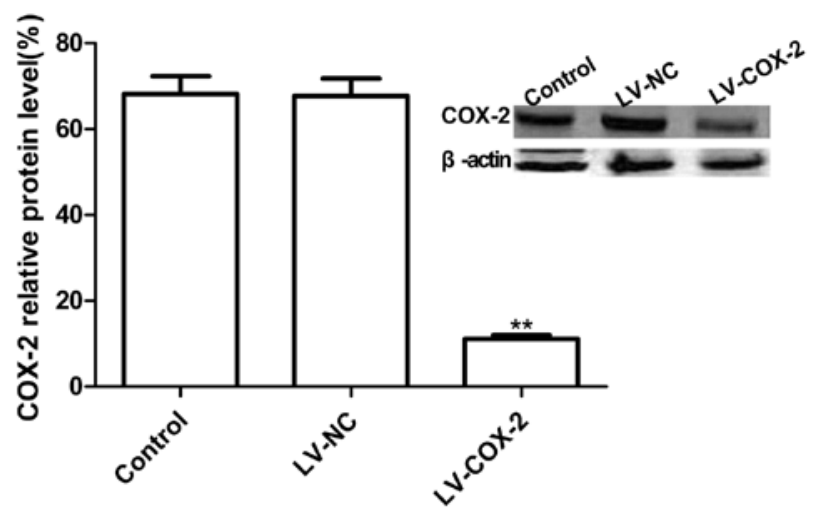

Figure 1. Silencing COX-2 by siRNA inhibits the COX-2 expression in MCF-7 tumor cells. (A) Quantitative real-time PCR analysis of COX-2 mRNA expression after RNAi silencing; (B) Western blot analysis of COX-2 protein expression after RNAi silencing. The images are representative results from three independent experiments. ${ }^{*} \mathrm{P}<0.05,{ }^{* *} \mathrm{P}<0.01$ vs. control.

inhibition in COX-2 protein expression found in LV-NC group and control group ( $\mathrm{P}>0.05)$, while the band density decreased dramatically in the LV-COX-2 group as compared with the LV-NC and control group $(\mathrm{P}<0.01)$ (Fig. 1B). These results demonstrated that LV-COX-2 significantly decreased COX-2 protein expression in the breast cancer cell line $(\mathrm{P}<0.01)$.

Effects of ATM and LV-COX-2 alone or combination on MCF-7 cell proliferation and the cell cycle. To evaluate the effect of LV-COX-2 and ATM alone and both on the cell viability of breast cancer cells in vitro, MTT assay was performed for $48 \mathrm{~h}$ when MCF-7 were treated with LV-COX-2 and ATM alone or combination. It was found that the inhibitory rates of LV-COX-2 and ATM alone or combination treatment were higher than control group and LV-NC treatment group $(\mathrm{P}<0.01)$. There is no significance different between LV-NC group and control group $(\mathrm{P}>0.05)$. In addition, the inhibitory rates of LV-COX-2 in combination with ATM group were higher than LV-COX-2 group and ATM treatment group (Fig. 2A).

The effects of LV-COX-2 and ATM on the cell cycles of MCF-7 cells were then analyzed by flow cytometry. MCF-7 cells treated with LV-COX-2 or ATM had an increased 
A

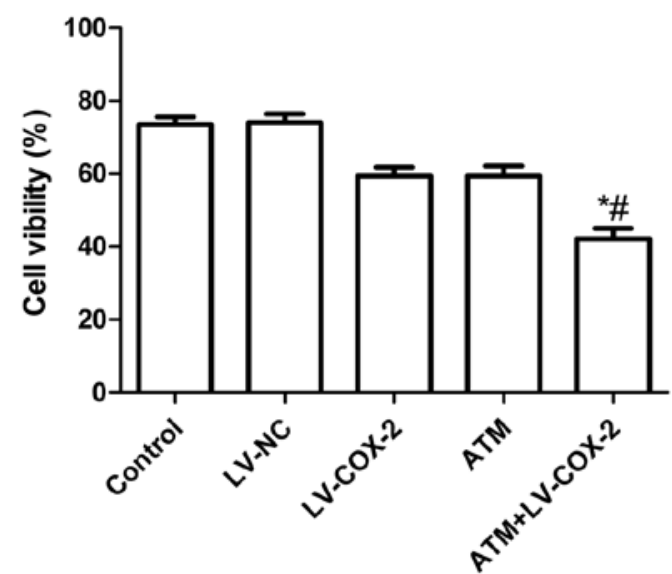

B

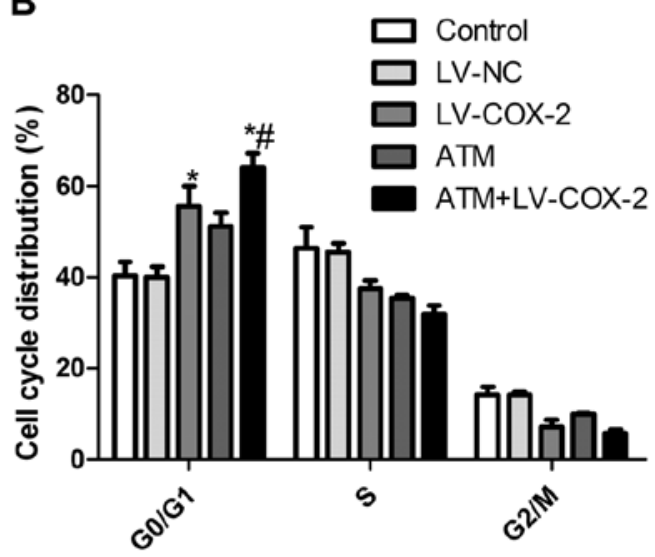

Figure 2. Effect of ATM and LV-COX-2 alone and in combination on cell proliferation and cell cycle of MCF-7 cells. Cell proliferation (A) and cell cycle (B) of MCF-7 cell was determined $48 \mathrm{~h}$ after treatment with ATM and LV-COX-2 alone or combination. ${ }^{*} \mathrm{P}<0.05$ versus control, ${ }^{*} \mathrm{P}<0.05$ versus ATM alone.

A

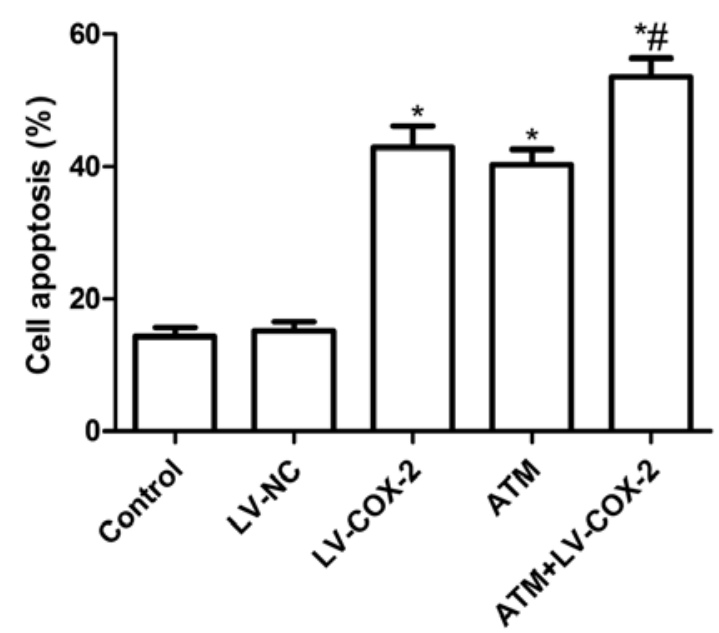

B

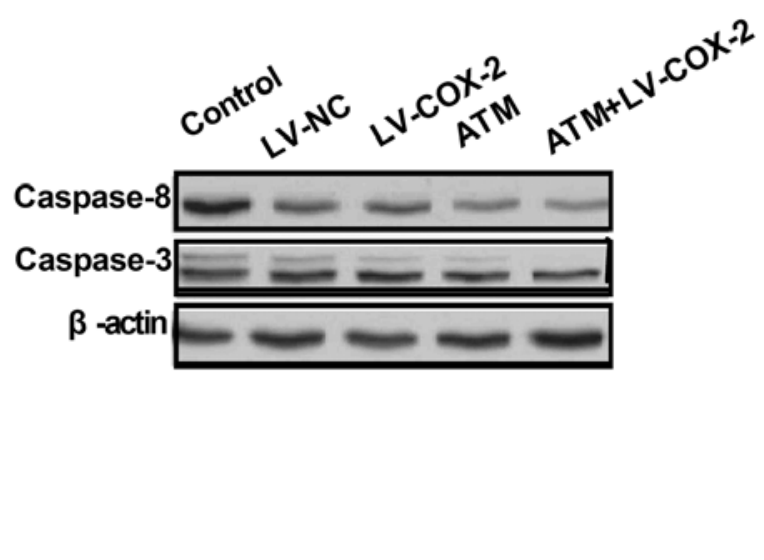

Figure 3. Effect of ATM and LV-COX-2 alone and in combination on cell apoptosis in MCF-7 cells. (A) Cell apoptosis of MCF-7 cell was determined 48 h after treatment with ATM and LV-COX-2 alone or combination; (B) Expression of caspase-3 and -8 in MCF-7 cell was determined by western blot analysis. Data are expressed as are the means $\pm \mathrm{SD} .{ }^{*} \mathrm{P}<0.01$ versus control, ${ }^{\#} \mathrm{P}<0.01$ versus ATM alone.

percentage of arrest at the G0/G1 phase compared with untreated cells and LV-NC control (Fig. 2B). The LV-COX-2 combination with ATM resulted in an even greater percentage of arrest at the G0/G1 phase than the higher doses of either drug alone $(\mathrm{P}<0.01)$.

Effects of ATM and LV-COX-2 alone or combination on MCF-7 cell apoptosis. To investigate whether the LV-COX-2 and ATM alone or combination could induce of apoptosis, we analyzed apoptosis after treatment with LV-COX-2 and ATM. It was found that MCF-7 cells treated with LV-COX-2 or ATM could significantly induce cell apoptosis compared with untreated cells and LV-NC control (Fig. 3A). Treatment with combination of LV-COX-2 and ATM led to a dramatic increase in apoptotic cells compare to single LV-COX-2 group and control group $(\mathrm{P}<0.01)$ (Fig. $3 \mathrm{~A})$.
To explore the possible mechanism of induction of cell apoptosis of combination with LV-COX-2 and ATM, expression patterns of caspase- 3 and -8 were determined by western blotting. The results showed that combination with LV-COX-2 and ATM could significantly decrease the expression of apoptosis inhibiting genes, caspase-3 and -8, in MCF-7 cells, compared to LV-COX-2 or ATM alone $(\mathrm{P}<0.01)$ (Fig. 3B).

Effects of ATM and LV-COX-2 on MCF-7 cell migration and cell invasion. To ascertain the inhibitory effect of TAM and LV-COX-2 as a single or combined treatment on breast cancer migration, wound-healing assay was performed to investigate their effects on the migration potential of MCF-7 cells. After 48-h treatment, cells in the control group and LV-NC group efficiently spread into the wound area to such an extent that the wound boundary was not apparent, while only some cells 
A
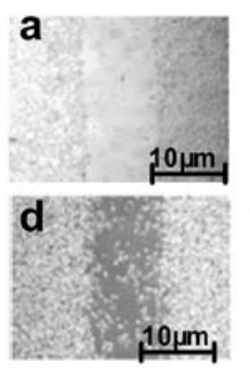
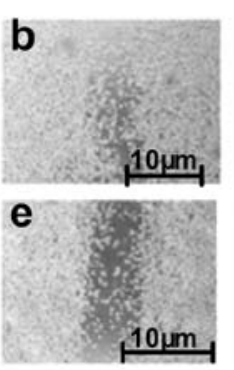
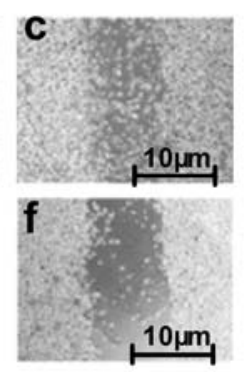

B

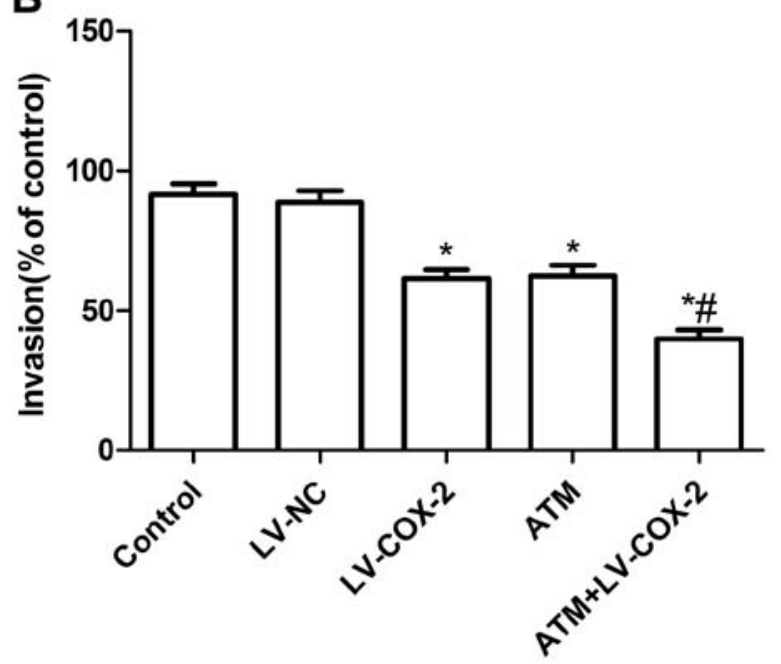

Figure 4. Effect of ATM and LV-COX-2 alone and in combination on cell invasion in MCF-7 cells. (A) Cell invasion was determined $48 \mathrm{~h}$ after treated with ATM and LV-COX-2 alone or combination; phase micrographs of cells were taken at 0 and $48 \mathrm{~h}$ after monolayer wounding. (a) Control group in 0 day; (b) Control group after 48 h; (c) MCF-7 cells treated with LV-NC; (d) MCF-7 cells treated with LV-COX-2; (e) MCF-7 cells treated with ATM; (f) MCF-7 cells treated with combination of LV-COX-2 and ATM. Results are the average of three independent experiments, in triplicates. (B) TAM and LV-COX-2 alone or combination both effect MCF-7 cell invasion through Matrigel. ${ }^{*} \mathrm{P}<0.01$ versus control, ${ }^{*} \mathrm{P}<0.01$ versus ATM alone.

A

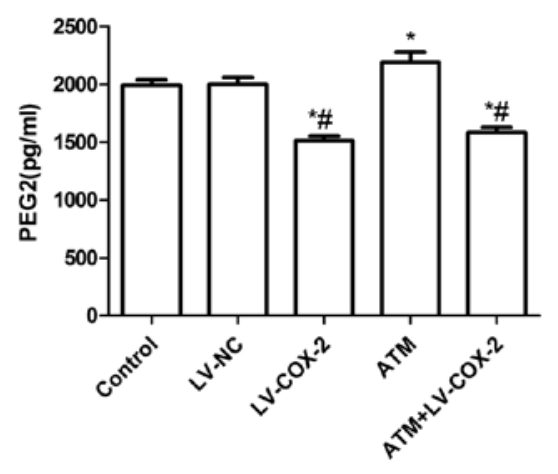

C

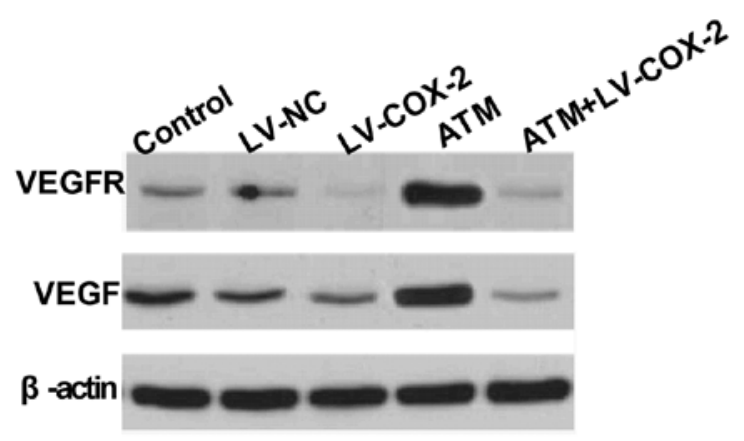

B
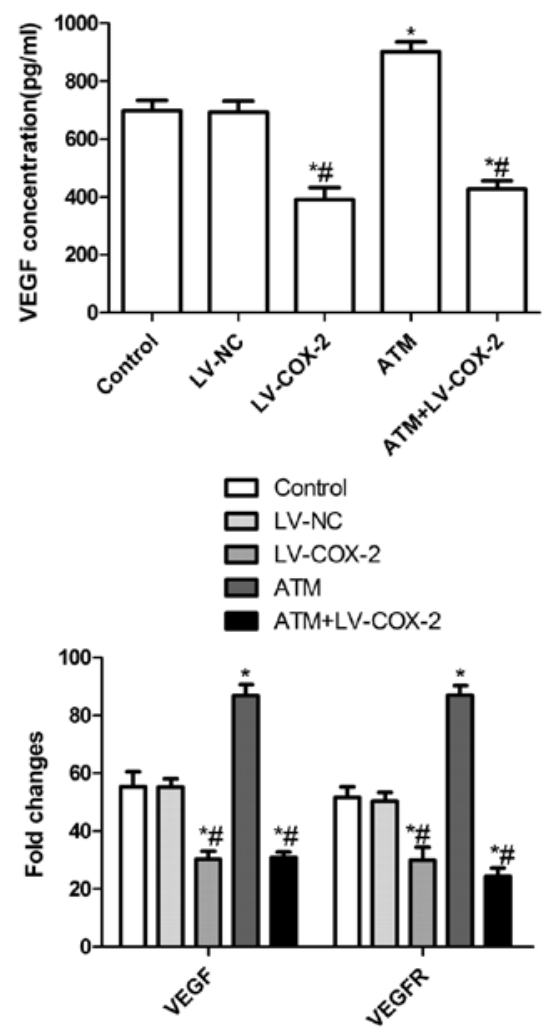

Figure 5. Effect of ATM and LV-COX-2 alone and in combination on PEG2 production and VEGF expression level in MCF-7 cells. Cells were treated with TAM, LV-COX-2, or both and incubated in serum-free conditioned medium for $24 \mathrm{~h}$. PEG2 production (A) and VEGF levels (B) were determined by ELISA. Expression of VEGF and VEGFR in MCF-7 cell was determined by western blot analysis (C and D). Data are expressed as the means \pm SD. ${ }^{*} \mathrm{P}<0.01$ versus control, ${ }^{\#} \mathrm{P}<0.01$ versus ATM alone.

in TAM or LV-COX-2 treated group spread forward in MCF-7 cells. The cell migration in combination group was less than either drug alone (Fig. 4A).
The ability of TAM and LV-COX-2 to reduce the invasiveness of MCF7 cells was further investigated by the transwell system assay. It was found that invasion was also decreased 

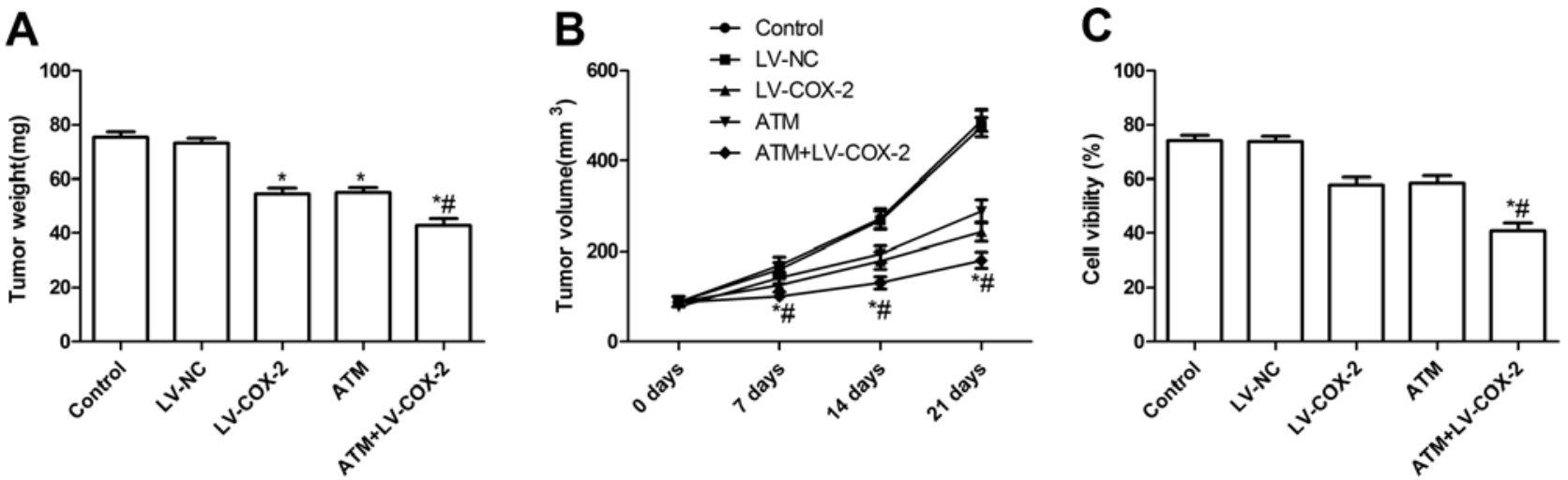

Figure 6. Antitumor activity of ATM and LV-COX-2 in BALB/c mice bearing MCF-7 cells. (A) Tumor weight in treated and untreated mice for 21 days (B) Tumor volume in treated and untreated mice at day 7, 14 and 21. (C) MTT assay of proliferation of splenocytes from mice. Data are expressed as the means $\pm \mathrm{SD} .{ }^{*} \mathrm{P}<0.01$ versus control, ${ }^{\#} \mathrm{P}<0.01$ versus $\mathrm{ATM}$ alone.

significantly with TAM or LV-COX-2 treatment compared to control and LV-NC $(\mathrm{P}<0.01)$ (Fig. 4B). Compared with the results with either agent alone, the combination of TAM and LV-COX-2 greatly inhibited MCF-7 cell invasion.

Effects of LV-COX-2 and ATM on PGE2 production and $V E G F$ expression level in MCF-7 cells. To examine the effect of ATM and LV-COX-2 alone or combination on PGE2 production in MCF-7 cells, ELISA was performed. As shown in Fig. 5A, LV-COX-2 single or combination with ATM inhibited PGE2 production. However, ATM increased PGE2 production compared to the other groups.

We also investigated the role of TAM and LV-COX-2 in the inhibition of secretory VEGF, a pro-angiogenic factor responsible for the migration and invasion of breast cancer cells. VEGF secretion in serum-free culture conditioned medium was assessed in MCF-7 cells by ELISA $24 \mathrm{~h}$ post-treatment. The results showed that TAM alone considerably upregulated VEGF secretion and the LV-COX-2 or LV-COX-2 combination TAM significantly decreased VEGF secretion compared with no treatment and LV-NC treatment (Fig. 5B).

To further study the possible mechanism of induction VEGF expression with LV-COX-2 and ATM, VEGF and VEGFR expression were determined by western blotting. The results showed that combination with LV-COX-2 and ATM or single LV-COX-2 could significantly decrease the expression of VEGF, VEGFR expression compared to control group and LV-NC group $(\mathrm{P}<0.01)$ (Fig. 5C and D). On the other hand, ATM dramatically increased the expression of VEGF, VEGFR expression compared to control group and LV-NC group $(\mathrm{P}<0.01)$ (Fig. $5 \mathrm{C}$ and $\mathrm{D})$. These results implied that combination with LV-COX-2 and ATM could inhibit PGE2 production and VEGF secretion by inhibition VEGF and VEGFR expression.

Antitumor activity of TAM and LV-COX-2 in nude mice bearing MCF-7 tumors. We assessed the in vivo therapeutic efficacy of TAM and LV-COX-2 in female BALB mice bearing MCF-7 breast tumors cells. Mice were sacrificed and tumor tissue harvested 21 days after treatment. Then tumor weight of the animals was measured. Tumor weight of LV-COX-2 and ATM alone or combination group was lower than those of control group and LV-NC group (Fig. 6A). Compared with the results with either agent alone, the combination of TAM and LV-COX-2 greatly inhibited tumor growth. In addition, we found that tumor volume after treatment with LV-COX-2 and ATM was significantly slower for MCF-7 tumor cells compared with control group and LV-NC group (Fig. 6B). Treatment with combination of LV-COX-2 and ATM led to a dramatic inhibition of tumor growth compared to single LV-COX-2 group and control group $(\mathrm{P}<0.01)$ (Fig. 6B). These results indicate that LV-COX-2 combination with ATM treatment in breast cancer cells markedly suppresses their tumorigenicity in mice.

We also assess the efficacy of LV-COX-2 combination with ATM in modulating splenocyte proliferation using MTT assay. As shown in Fig. 6C, the inhibitory rates of LV-COX-2 and ATM alone or combination significantly increased compared to control group and LV-NC group $(\mathrm{P}<0.01)$. The inhibitory rates of LV-COX-2 combination with ATM treatment group were higher than single drug groups, which demonstrated that combination treatment could inhibit MCF-7 cell proliferation.

\section{Discussion}

Estrogen can cause a general upregulation of genes regulating cell proliferation and survival and the downregulation of genes with anti-proliferative or pro-apoptotic activity resulting in growth stimulation and apoptosis suppression (25). Therefore, anti-estrogens are able to decrease cancer cell proliferation and induce cell death signaling pathways (26). Consequently, tamoxifen (TAM), a selective estrogen receptor (ER) modulator, has been described as 'the most important drug developed in the history of breast cancer' (27) and can induce cell cycle arrest leads to an accumulation of cancer cells in G0/G1 phase of the cell cycle (28) and induce apoptosis of breast cancer cells (29). However, a large number of initial clinical studies of TAM displayed its anti-angiogenic and VEGF reducing ability in various tumor models (30-33). In addition, an undesirable response leading to enhanced metastasis and angiogenesis and resulting in inferior outcomes, when prolonged administration of TAM 
causes intracellular VEGF levels to rise in patients (34). In this context, we made an attempt to decrease intracellular VEGF levels by decreasing ATM dose in breast cancer cells. Furthermore, recently studies showed that siRNA-mediated knockdown of COX-2 expression inhibited VEGF expression (35). Therefore, in this study, we knocked out COX-2 gene via a replication-incompetent lentivirus (LV-COX-2), then LV-COX-2 combination with TAM for reducing doses of ATM. The result showed that this novel combination inhibited COX-2 expression, downregulated the level of PGE2 and decreased the production of vascular endothelial growth factor (VEGF) in tumors.

A recent study showed that the combination of TAM and CXB at nontoxic levels exerts potent anti-angiogenic effects by decrease in VEGF expression (16), which was in agreement with our results. However, the celecoxib analgesia also faces the gastrointestinal side effects (36) and tolerance as observed in a rat model of inflammatory pain (37). Furthermore, celecoxib could result in liver injury $(17,18)$. These side effects would limit their clinical applications. In this study, we selected LV-COX-2 combiantion with ATM avoiding the side effects induced by celecoxib.

Extensive studies showed that shRNA and transgene carried by lentiviral vector can be transcribed into precursor mRNA where shRNA is embedded in the 3'UTR or in the intron of transgene (38-40). It has been shown that RNA combination anticancer drug could achieve better antitumor activity (21). Luni et al demonstrated that when melanoma differentiation associated gene-7 (mda-7) via a replicationincompetent adenovirus (Ad.mda-7) and gefitinib are used in combination they might provide an effective therapeutic approach for NSCLC since these agents target multiple cell survival pathways and are equally effective against NSCLC cells (41). In the present study, we used a novel strategy of combining a gene therapy approach, LV-COX-2, with ATM, and evaluated its antitumor effects against breast cancer cells. The result showed that LV-COX-2 combination with TAM in breast cancer cells significantly suppressed the proliferation and metastasis, and induced tumor apoptosis in vitro, and tumor growth also was suppressed in vivo.

In conclusion, our in vitro studies demonstrate that when LV-COX-2 and ATM are used in combination they might provide an effective therapeutic approach for breast cancer since these agents target induction breast cell apoptosis and are equally effective against breast cancer cells. Additionally, further studies with in vivo tumor models also confirmed that LV-COX-2 combination with ATM could suppress breast tumor growth. Therefore, it may be worthwhile to consider the combination treatment as novel therapeutic strategy for further evaluation in clinical trials.

\section{Acknowledgements}

This study was supported by Science and Technology Research and Innovation Team Fund of Jilin province (JL2011038).

\section{References}

1. Jordan VC and O'Malley BW: Selective estrogen-receptor modulators and antihormonal resistance in breast cancer. J Clin Oncol 25: 5815-5824, 2007.
2. Brauch $\mathrm{H}$ and Jordan VC: Targeting of tamoxifen to enhance antitumour action for the treatment and prevention of breast cancer: the 'personalised' approach? Eur J Cancer 45: 2274-2283, 2009.

3. Cuzick J, Sestak I, Pinder SE, et al: Effect of tamoxifen and radiotherapy in women with locally excised ductal carcinoma in situ: long-term results from the UK/ANZ DCIS trial. Lancet Oncol 12: 21-29, 2011.

4. Roberts CG, Millar EK, O'Toole SA, et al: Identification of PUMA as an estrogen target gene that mediates the apoptotic response to tamoxifen in human breast cancer cells and predicts patient outcome and tamoxifen responsiveness in breast cancer. Oncogene 30: 3186-3197, 2011.

5. Garvin S, Nilsson UW and Dabrosin C: Effects of oestradiol and tamoxifen on VEGF, soluble VEGFR-1, and VEGFR-2 in breast cancer and endothelial cells. Br J Cancer 93: 1005-1010, 2005.

6. Qu Z, Van Ginkel S, Roy AM, et al: Vascular endothelial growth factor reduces tamoxifen efficacy and promotes metastatic colonization and desmoplasia in breast tumors. Cancer Res 68: 6232-6240, 2008.

7. Majeti BK, Lee JH, Simmons BH and Shojaei F: VEGF is an important mediator of tumor angiogenesis in malignant lesions in a genetically engineered mouse model of lung adenocarcinoma. BMC Cancer 13: 213, 2013.

8. Chen CT and Hung MC: Beyond anti-VEGF: dual-targeting antiangiogenic and antiproliferative therapy. Am J Transl Res 5: 393-403, 2013.

9. Breinig M, Schirmacher P and Kern MA: Cyclooxygenase-2 (COX-2) - a therapeutic target in liver cancer? Curr Pharm Des 13: 3305-3315, 2007.

10. Stasinopoulos I, O'Brien DR, Wildes F, Glunde K and Bhujwalla ZM: Silencing of cyclooxygenase- 2 inhibits metastasis and delays tumor onset of poorly differentiated metastatic breast cancer cells. Mol Cancer Res 5: 435-442, 2007.

11. de Heer P, Sandel MH, Guertens G, et al: Celecoxib inhibits growth of tumors in a syngeneic rat liver metastases model for colorectal cancer. Cancer Chemother Pharmacol 62: 811-819, 2008.

12. Narayanan BA, Narayanan NK, Davis L and Nargi D: RNA interference-mediated cyclooxygenase- 2 inhibition prevents prostate cancer cell growth and induces differentiation: modulation of neuronal protein synaptophysin, cyclin D1, and androgen receptor. Mol Cancer Ther 5: 1117-1125, 2006.

13. Singh-Ranger G, Salhab M and Mokbel K: The role of cyclooxygenase-2 in breast cancer: review. Breast Cancer Res Treat 109: 189-198, 2008.

14. Hoeben A, Landuyt B, Highley MS, Wildiers H, Van Oosterom AT and De Bruijn EA: Vascular endothelial growth factor and angiogenesis. Pharmacol Rev 56: 549-580, 2004.

15. Hasegawa K, Ichikawa W, Fujita T, et al: Expression of cyclooxygenase-2 (COX-2) mRNA in human colorectal adenomas. Eur J Cancer 37: 1469-1474, 2001.

16. Kumar BN, Rajput S, Dey KK, et al: Celecoxib alleviates tamoxifen-instigated angiogenic effects by ROS-dependent VEGF/ VEGFR2 autocrine signaling. BMC Cancer 13: 273, 2013.

17. Lacroix I, Lapeyre-Mestre M, Bagheri H, et al: Nonsteroidal antiinflammatory drug-induced liver injury: a case-control study in primary care. Fundam Clin Pharmacol 18: 201-206, 2004.

18. Rudnick DA, Shikapwashya O, Blomenkamp K and Teckman JH: Indomethacin increases liver damage in a murine model of liver injury from alpha-1-antitrypsin deficiency. Hepatology 44: 976-982, 2006.

19. Park JW, Park JE, Lee JA, Lee CW and Kim CM: Cyclooxygenase-2 (COX-2) is directly involved but not decisive in proliferation of human hepatocellular carcinoma cells. J Cancer Res Clin Oncol 132: 184-192, 2006.

20. Wang R, Wang X, Lin F, Gao P, Dong K and Zhang HZ: shRNAtargeted cyclooxygenase (COX)-2 inhibits proliferation, reduces invasion and enhances chemosensitivity in laryngeal carcinoma cells. Mol Cell Biochem 317: 179-188, 2008.

21. Lin T, Gu J, Zhang L, et al: Enhancing adenovirus-mediated gene transfer in vitro and in vivo by addition of protamine and hydrocortisone. J Gene Med 5: 868-875, 2003.

22. Coleman JE, Huentelman MJ, Kasparov S, et al: Efficient largescale production and concentration of HIV-1-based lentiviral vectors for use in vivo. Physiol Genomics 12: 221-228, 2003.

23. Deglon N, Tseng JL, Bensadoun JC, et al: Self-inactivating lentiviral vectors with enhanced transgene expression as potential gene transfer system in Parkinson's disease. Hum Gene Ther 11: 179-190, 2000. 
24. Tai MH, Weng $\mathrm{CH}$, Mon DP, Hu CY and Wu MH: Ultraviolet $\mathrm{C}$ irradiation induces different expression of cyclooxygenase 2 in NIH 3T3 cells and A431 cells: the roles of COX-2 are different in various cell lines. Int J Mol Sci 13: 4351-4366, 2012.

25. Frasor J, Danes JM, Komm B, Chang KC, Lyttle CR and Katzenellenbogen BS: Profiling of estrogen up- and downregulated gene expression in human breast cancer cells: insights into gene networks and pathways underlying estrogenic control of proliferation and cell phenotype. Endocrinology 144: 4562-4574, 2003.

26. Renoir JM, Bouclier C, Seguin A, Marsaud V and Sola B: Antioestrogen-mediated cell cycle arrest and apoptosis induction in breast cancer and multiple myeloma cells. J Mol Endocrinol 40: 101-112, 2008

27. Zheng $\mathrm{J}$ and Yao Z: Effect of tamoxifen on apoptosis and drug resistance of breast cancer cells in vitro. Zhonghua Zhong Liu Za Zhi 22: 55-57, 2000 (In Chinese).

28. Thiantanawat A, Long BJ and Brodie AM: Signaling pathways of apoptosis activated by aromatase inhibitors and antiestrogens. Cancer Res 63: 8037-8050, 2003.

29. Rajput S, Kumar BN, Sarkar S, et al: Targeted apoptotic effects of thymoquinone and tamoxifen on XIAP mediated Akt regulation in breast cancer. PLoS One 8: e61342, 2013.

30. Blackwell KL, Haroon ZA, Shan S, et al: Tamoxifen inhibits angiogenesis in estrogen receptor-negative animal models. Clin Cancer Res 6: 4359-4364, 2000.

31. Marson LP, Kurian KM, Miller WR and Dixon JM: The effect of tamoxifen on breast tumour vascularity. Breast Cancer Res Treat 66: 9-15, 2001

32. Garvin S and Dabrosin C: Tamoxifen inhibits secretion of vascular endothelial growth factor in breast cancer in vivo. Cancer Res 63: 8742-8748, 2003.

33. McNamara DA, Harmey J, Wang JH, Kay E, Walsh TN and Bouchier-Hayes DJ: Tamoxifen inhibits endothelial cell proliferation and attenuates VEGF-mediated angiogenesis and migration in vivo. Eur J Surg Oncol 27: 714-718, 2001.
34. Ruohola JK, Valve EM, Karkkainen MJ, Joukov V, Alitalo K and Harkonen PL: Vascular endothelial growth factors are differentially regulated by steroid hormones and antiestrogens in breast cancer cells. Mol Cell Endocrinol 149: 29-40, 1999.

35. Liu H, Xiao J, Yang Y, et al: COX-2 expression is correlated with VEGF-C, lymphangiogenesis and lymph node metastasis in human cervical cancer. Microvasc Res 82: 131-140, 2011.

36. Mallen SR, Essex MN and Zhang R: Gastrointestinal tolerability of NSAIDs in elderly patients: a pooled analysis of 21 randomized clinical trials with celecoxib and nonselective NSAIDs. Curr Med Res Opin 27: 1359-1366, 2011.

37. Rezende RM, Paiva-Lima P, Dos Reis WG, Camelo VM, Bakhle YS and de Francischi JN: Celecoxib induces tolerance in a model of peripheral inflammatory pain in rats. Neuropharmacology 59: 551-557, 2010.

38. Du G, Yonekubo J,Zeng Y, Osisami M and Frohman MA: Design of expression vectors for RNA interference based on miRNAs and RNA splicing. FEBS J 273: 5421-5427, 2006.

39. Stegmeier F, Hu G, Rickles RJ, Hannon GJ and Elledge SJ: A lentiviral microRNA-based system for single-copy polymerase II-regulated RNA interference in mammalian cells. Proc Natl Acad Sci USA 102: 13212-13217, 2005.

40. Samakoglu S, Lisowski L, Budak-Alpdogan T, et al: A genetic strategy to treat sickle cell anemia by coregulating globin transgene expression and RNA interference. Nat Biotechnol 24: 89-94, 2006.

41. Emdad L, Lebedeva IV, Su ZZ, et al: Combinatorial treatment of non-small-cell lung cancers with gefitinib and Ad.mda-7 enhances apoptosis-induction and reverses resistance to a single therapy. J Cell Physiol 210: 549-559, 2007. 